\title{
Formation of Silver Nanoplates Layer on Amino Group Grafted Silica Coatings
}

\author{
Jurgis PILIPAVICIUS *, Ala CHODOSOVSKAJA, Aldona BEGANSKIENE, \\ Aivaras KAREIVA
}

\author{
Department of Inorganic Chemistry, Faculty of Chemistry, Vilnius University, Naugarduko 24, LT-03225 Vilnius, \\ Lithuania \\ cross $^{\text {ref }}$ http://dx.doi.org/10.5755/j01.ms.22.2.8405
}

Received 06 October 2014; accepted 31 March 2015

\begin{abstract}
In this study the self-arrangement of $\mathrm{Ag}$ nanoplates on (3-Aminopropyl)triethoxysilane (APTES) silanized silica coatings was investigated. Silica coatings were made by sol-gel method and silanized in two different ways. The first one includes silanization in acidic 2-propanol solution, the other one - in dry toluene. Coatings were silanized by using different amounts of APTES in case of silanization in 2-propanol. Silver nanoplates layer of functionalized silica coatings was obtained via self-assembly. Coatings were investigated by atomic force microscopy (AFM), water contact angle measurements (CA), FT-IR analysis, and scanning electron microscopy (SEM). Research showed that dense Ag nanoplates arrangement occurs when there is a high amount of amino groups on the surface.

Keywords: silica, sol-gel, APTES, silanization, silver nanoprisms.
\end{abstract}

\section{INTRODUCTION}

Nowadays nanostructured silica is one of the most extensively investigated material and has applications as optoelectronic devices [1], $\mathrm{CO}_{2}$ absorbents [2], photonic crystals [3], chemical/biological sensors [4], drug delivery [5]. Low cost synthesis, chemical versatility and stability of nanostructured silica makes it an ideal matrix for various composite materials. Silica derived by sol-gel method is widely used in formation of functional coatings. Chemically functionalized silica coatings are widely used as biomaterials [6], antireflective coatings [7,8], and for self-assembly of nanoparticles [9].

Spherical silver and gold nanoparticles and their properties were investigated by many researchers in 90's. In recent years, scientists are focused on anisotropic properties of silver and gold nanoparticles and their application in various fields $[10-12]$. Today we can find a lot information about the production of silver and gold nanocubes [13], nanododecahedron [14], nanodiscs [15] and nanoprisms [11]. Silver nanoprisms (AgNPRs) are one of the most interesting types of nanoparticles, because of its wide tuneability and high intensity of surface plasmon resonance (SPR) band $[16,17]$. By manipulating thickness and edge length of the particles, it is possible to shift SPR peak position in $400-1300 \mathrm{~nm}$ range, when conventional spherical particles exceeds only $600 \mathrm{~nm}$ [18]. These properties open the gateway for variety of applications in near-infrared technologies, such as telecommunications [18] or surface enhanced Raman spectroscopy (SERS) [19].

These particles are interesting because of their unique properties, however further details must be investigated on growth aspects and synthesis optimization. Nevertheless, it is very important to work on the compatibility with other materials and integration of those particles in other media.

\footnotetext{
* Corresponding author. Tel.: +370-5-2193184; fax: +370-5-2330987.

E-mail address: jurgis.pilipavicius@chf.vu.lt (J. Pilipavičius)
}

Therefore, our research is focused on synthesis of silicasilver nanoprism composite coatings. Currently, there is some available information about production of various types of composites with Ag NPRs. Most of them are based on incorporation of these particles into various polymer matrices $[20,21]$, but there were only few papers about the integration into the inorganic matrix. Our approach is to make silica-silver nanoprism coatings by self-assembly on functionalized silica surface. Xue, C., et al. [22, 23] has made the research about this topic, but there is no extensive study about the influence of different surface parameters to Ag nanoparticles self-assembly.

Thus this study is focused on the formation of silica coatings, different silanization techniques, and selfassembling of Ag nanoprisms on silanized surfaces. In this research we synthesized silica coatings by sol-gel method, silanized with (3-Aminopropyl)triethoxysilane (APTES) in two different techniques, and deposited layer of $\mathrm{Ag}$ nanoprisms by self-assembly. The process was investigated by atomic force microscopy (AFM), water contact angle measurements (CA), FT-IR analysis, and scanning electron microscopy (SEM).

\section{EXPERIMENTAL DETAILS}

\subsection{Chemicals and materials}

Tetraethyl orthosilicate (TEOS, $98 \%), \quad$ N- (3 Aminopropyl)triethoxysilane (APTES, $99 \%$ ), silver nitrate $\left(\mathrm{AgNO}_{3}, 99 \%\right)$, poly(sodium 4-styrenesulfonate) (PSSS, $1000000 \mathrm{Mw})$, sodium borohydride $\left(\mathrm{NaBH}_{4}, 99 \%\right)$, Lascorbic acid $\left(\mathrm{C}_{6} \mathrm{H}_{8} \mathrm{O}_{6}, 99 \%\right)$ were purchased from SigmaAldrich and used without further purification. Absolute ethanol $(99.9 \%)$ prepared by refluxing with preheated $\mathrm{CaO}$, and distilled after. Soda lime glass microscope slides $76 \times 26 \mathrm{~mm}$ (DG) were used as substrates in coating process, coatings made by using dip-coater (KSV Instruments DipCoater D). Hydrogen peroxide $\left(\mathrm{H}_{2} \mathrm{O}_{2}\right.$, $25 \%)$ and sulfuric acid $\left(\mathrm{H}_{2} \mathrm{SO}_{4} 98 \%\right)$ were used for substrate cleaning. 


\subsection{Synthesis of colloidal silica and coating procedure}

Colloidal silica sols in ethanol were made by acid catalyzed TEOS hydrolysis and condensation. For this purpose TEOS, $\mathrm{HCl}$, water and ethanol were mixed by molar ratio $1: 0.04: 4: 38$ respectively. Sols were left at room temperature for 5 hours.

The substrates were cleaned by immersing them in piranha solution (volume ratio $1: 4$ of $\mathrm{H}_{2} \mathrm{O}_{2}$ and $\mathrm{H}_{2} \mathrm{SO}_{4}$ respectively) for $15 \mathrm{~min}$. After cleaning, the substrates were washed with deionized water few times and dried by leaving in desiccator for $24 \mathrm{~h}$. Before coating procedure sols were filtered through $0.2 \mu \mathrm{m}$ Nylon filter to remove dusts and agglomerates. Coatings were made by DipCoating technique. All samples were prepared using the constant $40 \mathrm{~mm} / \mathrm{min}$ withdrawal speed. Coatings were dried for a few hours at room temperature in air atmosphere. Coated samples were ready for the next step surface silanization.

\subsection{Synthesis of silver NPRs}

Silver NPRs were made by seed-mediated method, which includes two steps - producing silver nanoseeds and growing nanoprisms.

Synthesis of silver seeds. In this experiment, silver seeds are obtained by mixing aqueous trisodium citrate $(20 \mathrm{~mL}, 25 \mathrm{mM})$, PSSS ( $1 \mathrm{ml}, 0.5 \mathrm{~g} / \mathrm{L})$, freshly prepared $\mathrm{NaBH}_{4}(1.2 \mathrm{~mL}, 10 \mathrm{mM})$ solutions, followed by addition of aqueous $\mathrm{AgNO}_{3}(20 \mathrm{~mL}, 0.5 \mathrm{mM})$. The addition rate of $\mathrm{AgNO}_{3}$ was $2 \mathrm{~mL} / \mathrm{min}$. The rate was maintained using syringe pump.

Growing NPRs. Silver NPRs are produced by mixing $35 \mathrm{ml}$ distilled water, aqueous ascorbic acid $(0.525 \mathrm{~mL}$, $10 \mathrm{mM}$ ) solution, $0.560 \mathrm{~mL}$ of prepared silver seeds solution, followed by addition of aqueous $\mathrm{AgNO}_{3}(21 \mathrm{~mL}$, $0.5 \mathrm{mM}$ ) at the rate of $1 \mathrm{~mL} / \mathrm{min}$. Solutions of obtained silver seeds and NPRs were characterized by UV-VIS spectroscopy (Perkin Elmer Lambda 35), scanning electron microscopy-electron dispersion spectroscopy (SEM/EDS, Hitachi FE-SEM SU-70).

\subsection{Surface silanization and deposition of AgNPRs}

Coatings were silanized by two methods - thermal silanization in dry toluene and in acidic 2-propanol solution.

Silanization in 2-propanol. Prepared silica coatings were immersed in 2-propanol solution of 1\% (v/v) APTES in presence of $0.1 \%$ acetic acid for $5 \mathrm{~h}$. The modified substrates were rinsed with pure 2-propanol, acetone and deionized water. Silanized coatings were dried 15 minutes in oven, at $120{ }^{\circ} \mathrm{C}$ temperature.

Silanization in toluene. Before silanization procedure toluene was dried by keeping under $3 \AA$ molecular sieves $(10 \% \mathrm{v} / \mathrm{w})$ and distilled after. Solutions of different concentrations of APTES in toluene were prepared. Then silica coatings were immersed in solution and refluxed for $12 \mathrm{~h}$ under argon atmosphere. After silanization, substrates were washed several times with pure toluene and acetone. Coatings were dried for 30 minutes at room temperature in air atmosphere. Silanized coatings were characterized by atomic force microscopy, FT-IR spectroscopy (Perkin Elmer FT-IR Frontier), and contact angle measurements (KSV Instruments CAM200).

For deposition of silver NPRs, functionalized substrates were immersed into further prepared silver NPRs colloidal solution and kept for 7 days. The composite coatings were gently washed with distilled water and left to dry at room temperature. Samples were characterized by UV-VIS absorption spectroscopy, SEM and evaluated visually.

\section{RESULTS AND DISCUSSION}

\subsection{Synthesis of colloidal silica, fabrication of coatings and surface silanization}

Two types of silica sols can be used for the amorphous silica coatings preparation - produced via acid and base catalyzed hydrolysis. As mentioned in experimental section, acid catalyzed silica sol was used in this work. Silica tends to form linear chains at low $\mathrm{pH}$ levels (acidic catalysis), and no particles are formed [24]. During the base catalyzed hydrolysis and condensation reactions, silica spheres are usually formed, and their diameter can be varied between nanometer and submicrometer. The exact size of the spheres is controlled by initial ratio of starting materials [25].

Silica coatings formed from silica nanoparticles have higher porosity and due to high surface area have more hydroxyl groups than coatings that are made from acid catalyzed silica sol. Therefore, during silanization process, silane molecules can be attached to the porous silica surface more efficiently.

However, surface roughness and porosity are hardly repeatable for coatings, obtained from base catalyzed than acid catalyzed sols. Therefore, it is more appropriate to use coatings with similar surface properties for the evaluation of two different silanization methods. Consequently, we selected acid catalyzed hydrolysis method for the preparation of sols.

After the substrates cleaning procedure, coatings were formed using dip-coating method. During this method film thickness is controlled by changing withdrawal speed. However, in our case film thickness does not play important role, so we have chosen medium, $40 \mathrm{~mm} / \mathrm{min}$ withdrawal speed for all samples. After the coating process, samples were kept for the next step - surface silanization.

In this study two silanization techniques were selected - silanization in dry toluene and in acidic 2-propanol solution. Both techniques are widely used for surface functionalization. In thermal silanization, dry non-polar solvents are refluxed in inert atmosphere together with alkoxysilane. Ideally, by this technique, only one layer of silane is formed on coating surface. Although this method is complex and due to toxicity it is hardly applicable in industry. The other method is performed at room temperature and it does not require toxic and extra dry solvents. In this technique substrates are kept in a mixture of alcohol, acid or base, water and desired alkoxysilane for few days. This method is simpler, but control of the thickness and homogeneity of silane layer is more complicated [26]. Thus we evaluated how differently 
silanized layer acts with silver NPRs. In this research we silanized coatings with both methods. In first method silica coatings were silanized by using $0.5 \%, 1 \%, 1.5 \%, 2 \%, 3 \%$ APTES in 2-propanol in presence of $0.1 \%$ acetic acid. In second method, coatings were silanized with $3 \%$ APTES in dry toluene.

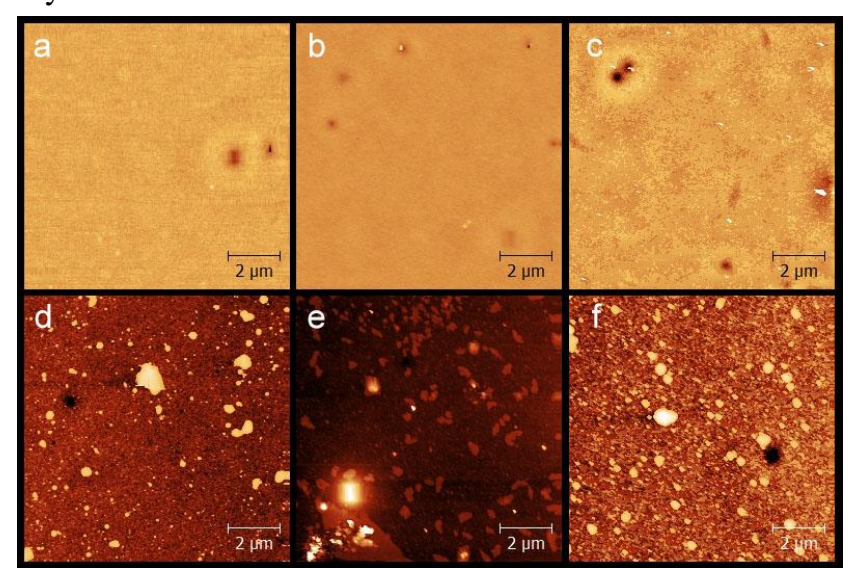

Fig. 1. AFM images of differently silanized silica coatings: $\mathrm{a}-0.5 \% ; \mathrm{b}-1 \% ; \mathrm{c}-1.5 \% ; \mathrm{d}-2 \%$; $-3 \%$ APTES in 2-propanol, $\mathrm{f}-3 \%$ APTES in toluene

After silanization, the obtained samples were investigated with AFM to evaluate the uniformity of obtained APTES layer (Fig. 1). From the AFM images it can be seen, that coatings silanized in 2-propanol forms uniform surface when APTES concentration is below $1.5 \%$. Coatings silanized with $2 \%$ and $3 \%$ APTES solutions no longer form the even surface, and micrometer size agglomerates are visible. Coatings silanized with $3 \%$ APTES in toluene are less uniform - submicrometer size agglomerates are observed. Additionally, for these samples water contact angles (CA) were measured. The calculated mean surface roughness and CA are presented in Table 1. As one can see from Table 1, calculated mean surface roughness correlates with $\mathrm{CA}$ values - increased surface roughness raises $\mathrm{CA}$ values.

Table 1. CA and surface roughness of silanized silica coatings

\begin{tabular}{|c|c|c|c|}
\hline Solvent & $\begin{array}{c}\text { APTES conc. in } \\
\text { the solvent, \% } \\
\text { (v/v) }\end{array}$ & $\begin{array}{c}\text { Contact } \\
\text { angle, } \\
\text { deg. }\end{array}$ & $\begin{array}{c}\text { Surface } \\
\text { roughness } R \mathrm{q}, \\
\mathrm{nm}\end{array}$ \\
\hline \multirow{3}{*}{ 2-Propanol } & $0.5 \%$ & $51 \pm 2$ & $0.2 \pm 0.2$ \\
\cline { 2 - 4 } & $1 \%$ & $56 \pm 2$ & $0.5 \pm 0.3$ \\
\cline { 2 - 4 } & $1.5 \%$ & $60 \pm 3$ & $0.4 \pm 0.3$ \\
\cline { 2 - 4 } & $2 \%$ & $65 \pm 4$ & $3.4 \pm 0.8$ \\
\hline Toluene & $3 \%$ & $59 \pm 4$ & $3.3 \pm 0.7$ \\
\hline
\end{tabular}

As one can see from the obtained results, coatings silanized in toluene have lowest uniformity and highest surface roughness. It is necessary to mention that latter silanization technique is very sensitive to purity of APTES and water content during silanization procedure [27]. Traces of water and/or presence of APTES oligomers can lead to self-polymerization of silane, and as result micrometer sized aggregates can form. In case of silanization in 2-propanol, higher content of APTES can also lead to rapid self-polymerization and formation of aggregates. Nevertheless, uniform coatings silanized in 2-propanol can be obtained if optimal amount of APTES is added.
This surface modification method is straightforward and well-studied, however silanized surface must be characterized to make sure, that surface functionalization took place. The presence of amine groups or attached hydrocarbon chains can be determined with FT-IR spectroscopy. Unfortunately, when mono or -bi layer of desirable functional groups forms on the surface, conventional FT-IR spectrometer equipped with ATR (Attenuated Total Reflectance) module hardly detects vibrations of these functional groups. Therefore, silanized silica coatings were formed on gold mirror to increase signal intensity.

FT-IR spectra of differently APTES silanized coatings can be seen in Fig. 2. Due to negligible difference of FT-IR spectra of coatings silanized in 2-propanol solution with various APTES concentration, only one (silanized by $1 \%$ APTES in 2-propanol) spectrum is presented.

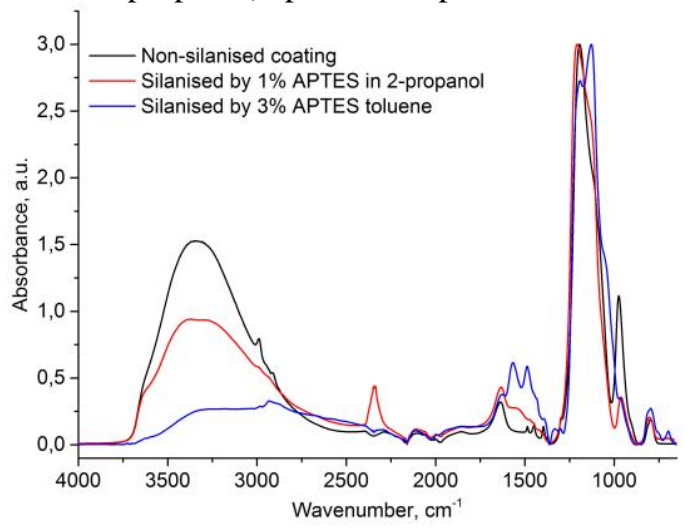

Fig. 2. Measured FT-IR spectra of silanized silica coatings

Vibrations at $1293 \mathrm{~cm}^{-1}$ are typical for the threedimensional -Si-O-Si- framework and the strong absorbance at $3338 \mathrm{~cm}^{-1}$ is from silica hydroxyl groups and absorbed water molecules. It is necessary to mention that the overlap of this peak with characteristic amine group vibrations in $3500-3300 \mathrm{~cm}^{-11}$ range makes FT-IR analysis more complicated. However, it is clear that the intensity of Si-OH peak is considerably lower in both silanized coatings. Although, another two characteristic medium intensity amine group peaks are observed at 1487 and $1567 \mathrm{~cm}^{-1}$, which are clearly visible in toluene silanized coating and small shoulder observable in 2-propanol silanized coating. The shoulder at $1128 \mathrm{~cm}^{-1}$ is slightly higher in both silanized samples, which also indicates primary amine group vibrations [28]. It can be concluded that coating modification performed in toluene leads to higher amount of the attached amine groups on the surface than silanized in 2-propanol.

\subsection{Synthesis of AgNPRs}

For the AgNPRs synthesis the seed-mediated method was selected, which was firstly suggested by Aherne, D., et al. [16]. By this method AgNPRs were synthesized in high yield, with edge length about $94 \pm 15 \mathrm{~nm}$. The average particle size was calculated form SEM micrographs (Fig. 3.) As can be seen from Fig. 3, the obtained particles have trigonal or hexagonal plate-like shape, but vary in size. The obtained colloidal AgNPRs solutions are relatively stable and no significant changes were observed in few months. 


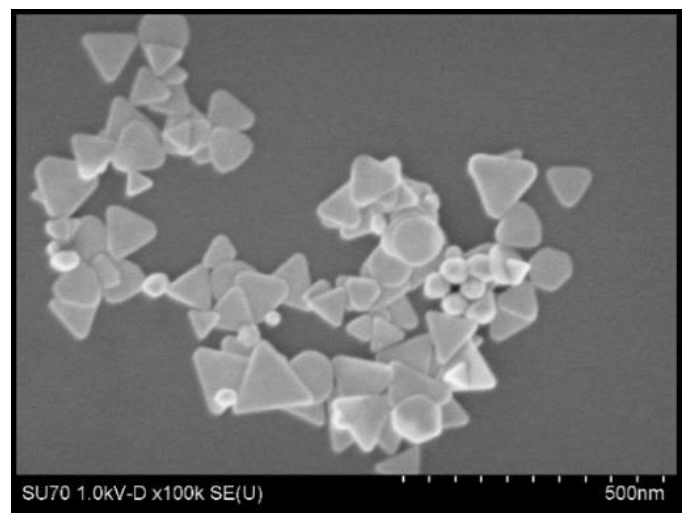

Fig. 3. SEM images of synthesized Ag NPR particles

The presented EDS spectrum (Fig. 4) shows elemental distribution in the measured sample. In SEM/EDS analysis silicon substrate was used, which gives high intensity $\mathrm{Si}$ peak. There is visible silver emission in EDS spectrum, which can be assigned to AgNPRs. Due to the presence of surfactants, carbon and oxygen peaks are also noticed in EDS spectrum.

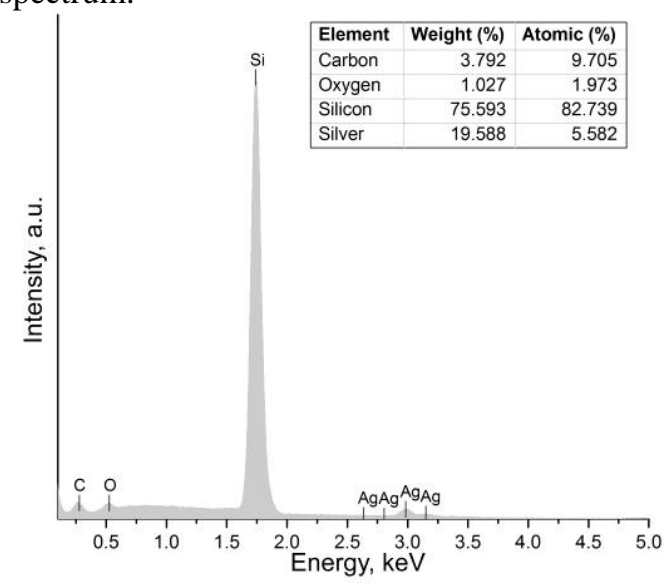

Fig. 4. EDS spectrum of synthesized Ag NPR's

Single SPR band (Fig. 5) shows high intensity absorption at $921 \mathrm{~nm}$, which gives a blue color of AgNPRs colloidal solution.

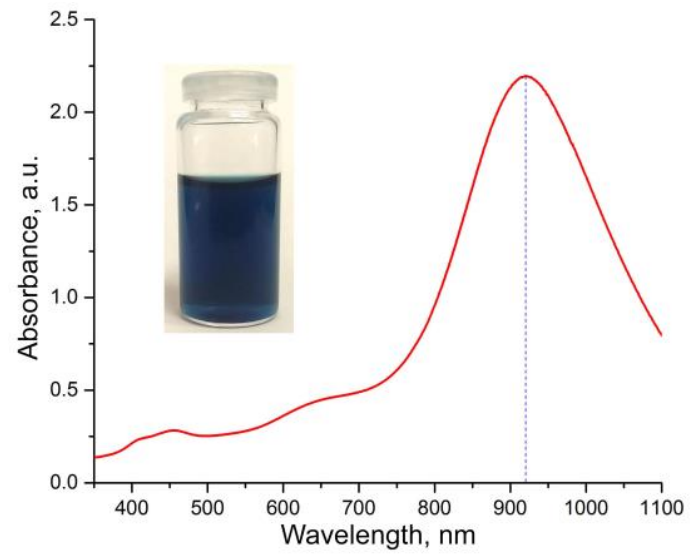

Fig. 5. Absorption spectra of synthesized Ag NPRs solution

Red shifted SPR peak indicates presence of high aspect ratio anisotropic triangular AgNPRs or nanohexagonal particles in the solution. It can also be seen that SPR peak is slightly broadened, but it is common for all the plasmonic particles that have absorption in near infrared [10].

\subsection{AgNPRs self-assembly on silanized surfaces}

Further we investigated AgNPRs self-assembly on differently silanized silica coatings. We tried to find out which silanized surface is more suitable for assembly of AgNPRs. Considering obtained surface uniformity of coatings, we selected coatings silanized by $0.5 \%, 1 \%$ $1.5 \%$ APTES solution in 2-propanol and $3 \%$ APTES in toluene. As mentioned in the experimental section, in order to obtain the self-assembled AgNPRs layer, functionalized coatings were vertically immersed in the plastic vials containing AgNPRs solution and kept for 1 week at room temperature. After this procedure, samples were ready for SEM analysis.

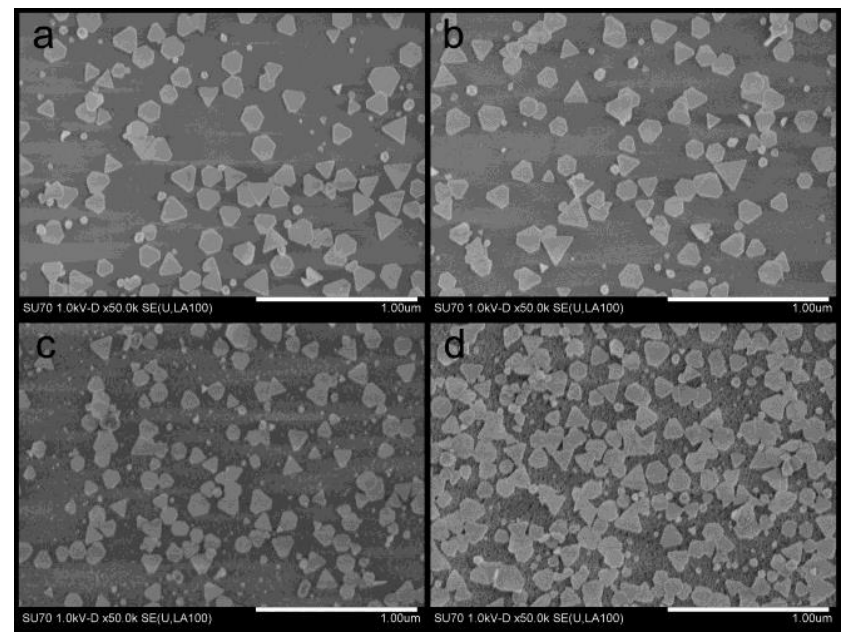

Fig. 6. SEM images of silanized and Ag NPRs deposited silica coatings: $\mathrm{a}-0.5 \% ; \mathrm{b}-1 \% ; \mathrm{c}-1.5 \%$ APTES in 2-propanol; $\mathrm{d}-3 \%$ APTES in toluene

As one can see from obtained SEM images (Fig. 6) uniformly deposited layer of AgNPRs is visible on all silanized coatings. The density of arranged AgNPRs layer is very similar on all coatings silanized in 2-propanol. Coatings silanized in toluene have denser distribution of AgNPRs. FT-IR analysis showed that coatings silanized in toluene have higher amount of amino groups than in 2-propanol silanized samples. We believe that silver nanoparticles have denser arrangement in coatings silanized in toluene due to higher amount of grafted APTES groups on the surface. However, silica coatings silanized in toluene are considerably less uniform than coatings silanized in 2-propanol. Contrary to our expectations, surface non-uniformity does not reduce the amount of deposited AgNPRs. The non-uniformity of coatings modified in toluene increases light scattering, which makes them less attractive for applications like SERS or optical devices.

Thus, coatings silanized in 2-propanol are more uniform, silanization procedure is simple and does not require hazardous solvents. Moreover, AgNPRs layer can be deposited via self-assembly in sufficient surface coverage. These advantages make them promising candidates for straightforward production of SERS substrates or optical components. 


\section{CONCLUSIONS}

In this brief study we successfully synthesized silica coatings by sol-gel method, functionalized with amino groups and deposited layer of AgNPRs. Most uniform silanized coatings were obtained by silanization in 2-pronanol, when amount of APTES is below $2 \%$. The highest amount of amino groups was obtained by silanization in toluene. However, AFM analysis shows non-uniform layer of silica when silanization in toluene is carried out. Nevertheless, the highest surface coverage with AgNPRs was obtained on coatings silanized in toluene. In the case of coatings silanized in 2-propanol, sell-assembled AgNPRs layer is less dense, but proper uniformity of coatings and straightforward silanization process make them more applicable as substrates for quantitative SERS analysis or optical materials.

\section{Acknowledgments}

Postdoctoral fellowship of A.C. is being funded by European Union Structural Funds project "Postdoctoral Fellowship Implementation in Lithuania" (No. SF-PD2012-12-31-0397).

\section{REFERENCES}

1. Kim, H.J., Choi, H.J., Nam, S.-M., Song, Y.W. High-Performance Laser Mode-Locker with Glass-Hosted Swnts Realized by RoomTemperature Aerosol Deposition Optical Express 19 (5) 2011: pp. $4762-4767$.

http://dx.doi.org/10.1364/OE.19.004762

2. Cui, S., Cheng, W., Shen, X., Fan, M., Russell, A., Wu, Z., Yi, X. Mesoporous Amine-Modified SiO2 Aerogel: A Potential CO2 Sorbent Energy \& Environmental Science 4 (6) 2011: pp. $2070-2074$.

http://dx.doi.org/10.1039/c0ee00442a

3. Stein, A., Schroden, R.C. Colloidal Crystal Templating of ThreeDimensionally Ordered Macroporous Solids: Materials for Photonics and Beyond Current Opinion in Solid State and Materials Science 5 (6) 2001: pp. 553-564.

http://dx.doi.org/10.1016/S1359-0286(01)00022-5

4. Cesarino, I., Marino, G., Matos, J. D. R., Cavalheiro, É.T.G. Evaluation of a Carbon Paste Electrode Modified with Organofunctionalised Sba-15 Nanostructured Silica in the Simultaneous Determination of Divalent Lead, Copper and Mercury Ions Talanta 75 (1) 2008: pp. 15-21.

http://dx.doi.org/10.1016/j.talanta.2007.06.032

5. Xiao, D., Jia, H. Z., Zhang, J., Liu, C. W., Zhuo, R. X., Zhang, X.Z. A Dual-Responsive Mesoporous Silica Nanoparticle for Tumor-Triggered Targeting Drug Delivery Small 10 (3) 2014: pp. 591-598.

6. Arcos, D., Vallet-Regí, M. Sol-Gel Silica-Based Biomaterials and Bone Tissue Regeneration Acta Biomaterialia 6(8) 2010: pp. 2874-2888.

7. Meng, X., Wang, Y., Wang, H., Zhong, J., Chen, R. Preparation of Hydrophobic and Abrasion-Resistant Silica Antireflective Coatings by Using a Cationic Surfactant to Regulate Surface Morphologies Solar Energy 101 2014: pp. 283-290.

8. Pilipavicius, J., Kazadojev, I., Beganskiene, A., Melninkaitis, A., Sirutkaitis, V., Kareiva, A. Hydrophobic Antireflective Silica Coatings via Sol-Gel Process Materials Science-Medziagotyra 14 (4) 2008: pp. 283-287.

9. An, Y., Chen, M., Xue, Q., Liu, W. Preparation and Self-Assembly of Carboxylic Acid-Functionalized Silica Journal of Colloid and Interface Science 311 (2) 2007: pp. 507-513.

http://dx.doi.org/10.1016/j.jcis.2007.02.084

10. Xiao, J., Qi, L. Surfactant-Assisted, Shape-Controlled Synthesis of Gold Nanocrystals Nanoscale 3 (4) 2011: pp. 1383-1396.
11. Millstone, J.E., $\quad$ Hurst, S.J., $\quad$ Métraux, G.S., Cutler, J.I., Mirkin, C.A. Colloidal Gold and Silver Triangular Nanoprisms Small 5 (6) 2009: pp. 646-664.

12. Cortie, M.B., McDonagh, A.M. Synthesis and Optical Properties of Hybrid and Alloy Plasmonic Nanoparticles Chemical Reviews 111 (6) 2011: pp. 3713-3735.

13. Kundu, S., Panigrahi, S., Praharaj, S., Basu, S., Ghosh, S.K., Pal, A., Pal, T. Anisotropic Growth of Gold Clusters to Gold Nanocubes under UV Irradiation Nanotechnology 18 (7) 2007: pp. 075712-075719.

14. Wang, D.W., Liu, Y., You, T.Y. A New Shape of Gold Nanocrystals: Singly Twinned Squashed Dodecahedron Crystengcomm 12 (12) 2010: pp. 4028-4030. http://dx.doi.org/10.1039/c0ce00096e

15. Jayabalan, J., Singh, A., Chari, R., Oak, S.M. Ultrafast ThirdOrder Nonlinearity of Silver Nanospheres and Nanodiscs Nanotechnology 18 (31) 2007: pp. 315704-315710.

16. Aherne, D., Ledwith, D. M., Gara, M., Kelly, J. M. Optical Properties and Growth Aspects of Silver Nanoprisms Produced by a Highly Reproducible and Rapid Synthesis at Room Temperature Advanced Functional Materials 18 (14) 2008: pp. 2005-2016.

17. Métraux, G.S., Mirkin, C.A. Rapid Thermal Synthesis of Silver Nanoprisms with Chemically Tailorable Thickness Advanced Materials 17 (4) 2005: pp. 412-415.

18. Bastys, V., Pastoriza-Santos, I., $\quad$ Rodríguez-González, B., Vaisnoras, R., Liz-Marzán, L.M. Formation of Silver Nanoprisms with Surface Plasmons at Communication Wavelengths Advanced Functional Materials 16 (6) 2006: pp. 766-773.

19. Yi, Z., Xu, X., Wu, X., Chen, C., Li, X., Luo, B., Luo, J., Jiang, X., Wu, W., Yi, Y., Tang, Y. Silver Nanoplates: Controlled Preparation, Self-Assembly, and Applications in Surface-Enhanced Raman Scattering Applied Physics A 110 (2) 2013: pp. 335-342. http://dx.doi.org/10.1007/s00339-012-7256-0

20. Berta, M., Loppinet, B., $\quad$ Vlassopoulos, D., Askounis, A., Koutsos, V., Pastoriza-Santos, I., Liz-Marzan, L.M. Tailoring the Properties of Grafted Silver Nanoprism Composites Polymer 53 (25) 2012: pp. 5771 - 5778.

21. Salvador, M., MacLeod, B.A., Hess, A., Kulkarni, A.P., Munechika, K., $\quad$ Chen, J. I. L., $\quad$ Ginger, D. S. Electron Accumulation on Metal Nanoparticles in Plasmon-Enhanced Organic Solar Cells Acs Nano 6 (11) 2012: pp. 10024-10032. http://dx.doi.org/10.1021/nn303725v

22. Xue, C., Chen, X., Hurst, S. J., Mirkin, C.A. Self-Assembled Monolayer Mediated Silica Coating of Silver Triangular Nanoprisms Advanced Materials 19 (22) 2007: pp. 4071-4074

23. Xue, C., Li, Z., Mirkin, C. A. Large-Scale Assembly of SingleCrystal Silver Nanoprism Monolayers Small $1(5)$ 2005: pp. 513-516.

24. Boonstra, A. H., Bernards, T. N. M. Hydrolysis-Condensation Reactions in the Acid Step of a Two-Step Silica Sol-Gel Process, Investigated with $29 \mathrm{si} \mathrm{Nmr}$ at $-75^{\circ} \mathrm{C}$ Journal of Non-Crystalline Solids 108 (3) 1989: pp. 249-259. http://dx.doi.org/10.1016/0022-3093(89)90295-0

25. Stöber, W., Fink, A., Bohn, E. Controlled Growth of Monodisperse Silica Spheres in the Micron Size Range Journal of Colloid and Interface Science 26 (1) 1968: pp. 62-69.

26. Guha Thakurta, S., Subramanian, A. Fabrication of Dense, Uniform Aminosilane Monolayers: A Platform for Protein or Ligand Immobilization Colloids and Surfaces A: Physicochemical and Engineering Aspects 414 2012: pp. 384-392. http://dx.doi.org/10.1016/j.colsurfa.2012.08.049

27. Zhu, M., Lerum, M. Z., Chen, W. How to Prepare Reproducible, Homogeneous, and Hydrolytically Stable Aminosilane-Derived Layers on Silica Langmuir 28 (1) 2011: pp. 416-423.

28. Zub, Y. L., Stolyarchuk, N. V., Barczak, M., Dąbrowski, A. Surface Heterogeneity of Polysiloxane Xerogels Functionalized by 3-Aminopropyl Groups Applied Surface Science 256 (17) 2010: pp. $5361-5364$ http://dx.doi.org/10.1016/j.apsusc.2009.12.079 